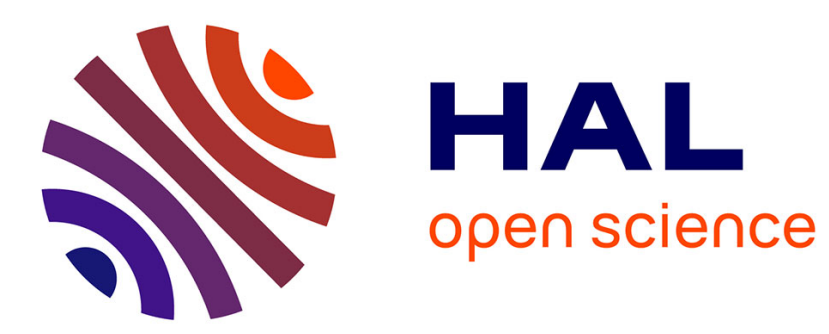

\title{
Player control of 'brassiness' at intermediate dynamic levels in brass instruments
}

Lisa Norman, John Chick, Murray Campbell, Arnold Myers, Joël Gilbert

\section{To cite this version:}

Lisa Norman, John Chick, Murray Campbell, Arnold Myers, Joël Gilbert. Player control of 'brassiness' at intermediate dynamic levels in brass instruments. 2010. hal-00474648

\section{HAL Id: hal-00474648 \\ https://hal.science/hal-00474648}

Preprint submitted on 21 Apr 2010

HAL is a multi-disciplinary open access archive for the deposit and dissemination of scientific research documents, whether they are published or not. The documents may come from teaching and research institutions in France or abroad, or from public or private research centers.
L'archive ouverte pluridisciplinaire HAL, est destinée au dépôt et à la diffusion de documents scientifiques de niveau recherche, publiés ou non, émanant des établissements d'enseignement et de recherche français ou étrangers, des laboratoires publics ou privés. 


\section{Editorial Manager(tm) for Acta Acustica united with Acustica}

Manuscript Draft

Manuscript Number: AAA-D-09-00113R2

Title: Player control of 'brassiness' at intermediate dynamic levels in brass instruments

Article Type: Special Issue Article

Corresponding Author: Mr Joel Gilbert,

Corresponding Author's Institution:

First Author: Lisa Norman

Order of Authors: Lisa Norman; John P Chick; Murray Campbell; Arnold Myers; Joel Gilbert

Abstract: An increase in the dynamic level of a brass instrument from 'forte' to 'fortissimo' generally brings about a change in timbre to a much brighter sound, often described as "brassy". This phenomenon is due to non-linear wave propagation along the bore of the instrument, which generates significant spectral enrichment in the higher harmonics. The dynamic level at which this change occurs is not always clearly defined and some brass players have found that, by employing slight changes in embouchure, they have a degree of control over the level of brassiness at a constant dynamic.

Experimental data from playing tests are presented, showing that players employing this technique are able to modify the gradient of the input pressure wave front as it is formed in the mouthpiece.

Numerical simulations based on weakly non-linear shock theory, with experimental data from brassy and non-brassy mouthpiece measurements as the input boundary conditions, confirm the assumption that the increase in spectral enrichment is primarily a consequence of enhancement of non-linear wave steepening resulting from a change in shape of the wave form in the mouthpiece.

Suggested Reviewers:

Opposed Reviewers: 


\title{
Player control of 'brassiness' at intermediate dynamic levels in brass instruments
}

\author{
L. Norman ${ }^{1}$, J.P. Chick ${ }^{2}$, D.M. Campbell ${ }^{3}$, A. Myers ${ }^{1}$ and J. Gilbert ${ }^{4}$ \\ ${ }^{1}$ School of Arts Culture and the Environment, University of Edinburgh, UK \\ ${ }^{2}$ School of Engineering, University of Edinburgh, UK \\ ${ }^{3}$ School of Physics, University of Edinburgh, UK \\ ${ }^{4}$ Laboratoire d'Acoustique de L'Université du Maine, Le Mans, France
}

February 4, 2010

\begin{abstract}
Summary
An increase in the dynamic level of a brass instrument from forte to fortissimo generally brings about a change in timbre to a much brighter sound, often described as 'brassy'. This phenomenon is due to non-linear wave propagation along the bore of the instrument, which generates significant spectral enrichment in the higher harmonics. The dynamic level at which this change occurs is not always clearly defined and some brass players have found that, by employing slight changes in embouchure, they have a degree of control over the level of brassiness at a constant dynamic. Experimental data from playing tests are presented, showing that players employing this technique are able to modify the gradient of the input pressure wave front as it is formed in the mouthpiece. Numerical simulations based on weakly nonlinear shock theory, with experimental data from brassy and non-brassy mouthpiece measurements as the input boundary conditions, confirm the assumption that the increase in spectral enrichment is primarily a consequence of enhancement of nonlinear wave steepening resulting from a change in shape of the waveform in the mouthpiece.
\end{abstract}

\section{Introduction}

One of the most striking characteristics of brass instruments is their wide dynamic range and the change in timbre that occurs from quiet to loud playing. At the loudest dynamic levels the tone of the instrument becomes typically brassy or cuivré. This is as a direct result of non-linear propagation of the pressure wave as it travels along the cylindrical sections of the bore of the instrument [1], causing the higher frequency components of the sound to be strongly excited and thus generating a bright and brilliant timbre. This style of playing is achieved most easily at the highest dynamic levels where non-linear 
propagation dominates. However, non-linear propagation of the wave front is not confined to the extremes of loud playing. It has also been observed to some extent at intermediate dynamic levels. Interestingly, brass players often talk about playing with or without a brassy 'edge' to the sound, but in reality the transition between a non-brassy and brassy timbre is not well defined [2].

The dominant factor influencing the timbre of the note is the bore profile [3]. However, mouthpiece dimensions [4], and construction material of the instrument [5][6] have also been cited as affecting the timbre. The issue of player control of brassiness through embouchure manipulation, the focus of this paper, is an area which has received little attention. Whereas the physical changes to the instrument can be measured in a relatively objective manner, the very nature and complexity of a player's lips and vocal tract make systematic measurement and analysis of variation in playing technique more difficult.

It is interesting to observe that player control of brassiness is much more easily achieved in brass instruments than in woodwind instruments. This is because brass instruments are generally more receptive to embouchure control than those in the woodwind family since properties of the reed (predominantly its stiffness) in an instrument such as the clarinet or oboe, are more or less fixed, whereas the brass player has a greater degree of control over the lips by changing the tension in the lip muscles, and the pressure and angle of the mouthpiece [7].

In Section 2 of this paper we present a summary of the underlying physical principles which determine the onset of non-linear propagation in brass instruments, and point to some of the possibilities that may be available to the player to control the spectral content of the sound. Section 3 documents an experimental procedure for assessing player control of the onset of brassiness in a french horn, and examines the results from playing tests. A numerical model of the instrument used for these experiments is described in Section 4 , and the output from this is used to show the separate contributions of linear and non-linear propagation to the spectral content of the radiated sound.

\section{Brightness and brassiness: theoretical background}

\subsection{Factors affecting spectral enrichment}

An elementary physical model capable of explaining the generation of sound by a brass instrument is illustrated in Figure 1. The lips of the player are represented as a valve modulating the flow of air from the player's lungs into the instrument. For a suitable choice of parameters the system behaves as a positive feedback loop, generating a selfsustaining oscillation [8]. The volume flow rate $q_{i n}$ through the valve is related to the acoustic pressure $p_{i n}$ in the mouthpiece of the instrument by a non-linear equation (Eq. 1) of the form:

$$
q_{i n}(t)=S(t) \sqrt{\frac{2\left(P_{M}-p_{i n}(t)\right)}{\rho}}
$$


where $S(t)$ is the area of the opening between the lips, $P_{M}$ is the pressure in the player's mouth, and $\rho$ is the density of air. The non-linear nature of Equation 1 has the important consequence that even if the players lips were to open and close sinusoidally, the air flow into the mouthpiece would have a non-sinusoidal waveform. The generation of upper harmonics is an inherent feature of the flow control valve, and the richness of the harmonic spectrum in the mouthpiece waveform increases with the pressure amplitude.

In fact, $S(t)$ does not normally have a strictly sinusoidal waveform. Studies of lip motion in brass players using transparent mouthpieces have also shown that the relationship between the opening height of the lips and the open area is typically non-linear, and it has been demonstrated that this non-linearity can contribute significantly to the spectral enrichment of the resulting sound [9].

A further factor which makes a significant contribution to the brightness of timbre in brass instruments is the high-pass filter effect of radiation from the bell of the instrument. A harmonic component at a low frequency $\omega$ can be considered as being radiated by a monopole source whose strength $Q_{\text {out }}$ is proportional to the pressure amplitude of the standing wave at frequency $\omega$ in the resonator. The resulting radiation far field is described by

$$
p_{\text {rad }}=\frac{j \omega Q_{\text {out }} \rho_{0}}{4 \pi r} \exp j(\omega t-k r)
$$

where $k$ is the wave number, and it is assumed that $1 / k$ is much larger than the bell radius. The dependence of the amplitude on $\omega$ implies a $6 \mathrm{~dB} /$ octave treble boost in the transfer function from internal to radiated pressure. Above the bell cutoff frequency, which is typically around $1.5 \mathrm{kHz}$ for a trumpet, the transfer function is close to unity and independent of frequency [10].

The model described so far includes a localised non-linear element in the flow control valve, but assumes that the behaviour of the sound waves in the resonating air column and in the radiation field can be described using linear acoustics. However, this model is incapable of explaining the dramatic increase in the relative amplitudes of the upper harmonics which occurs in very loud playing. This is due to non-linear propagation of the sound waves travelling from the mouthpiece to the bell. For fortissimo playing the pressure amplitude in the mouthpiece of a brass instrument can be of the order of $10 \mathrm{kPa}$; the linear acoustic assumption of constant sound propagation velocity is then inadequate. For the weakly non-linear case, the propagation speed $c$ is given by [11]

$$
c=c_{o}+\left(\frac{\gamma+1}{2}\right) v .
$$

where $c_{o}$ is the propagation speed in the linear approximation, $\gamma$ is the ratio of specific heats and $v$ is the acoustic particle velocity. The implication of Equation 3 is that, for the part of the acoustic cycle in which $v$ is positive, the speed of propagation increases with amplitude. This can lead to the higher parts of the wave catching up with the lower parts, steepening the rising wavefront, and eventually giving rise to a shock wave. 
For a cylindrical pipe, the distance $x_{s}$ which the wave travels before the shock wave is fully developed is given by the expression

$$
x_{s}=\frac{2 \gamma P_{a t} c_{o}}{(\gamma+1)\left(d p_{i n} / d t\right)_{\max }}
$$

where $P_{a t}$ is atmospheric pressure. The distance to shock formation thus depends inversely on the maximum value of the rate of change of the input pressure.

A brass instrument like a trombone has a substantial cylindrical section and then a section where the bore expands towards the bell. In the expanding section the rate of steepening of the travelling wave is diminished because the sound energy is distributed over an increasingly large cross-section, thereby reducing the acoustic velocity. This effect can be taken into account by introducing the coordinate stretching function $z(L)$ :

$$
z(L)=\int_{0}^{L} \frac{D(0)}{D(y)} d y
$$

where $D(0)$ is the diameter at the input, $D(y)$ is the diameter at a distance $y$ along the axis of the tube, and $L$ is the overall length of the instrument. $z(L)$ is the length of the cylindrical tube of diameter $D(0)$ which has the same degree of non-linear steepening as the instrument in question.

\subsection{Player control of timbral enrichment}

The question addressed in this paper is the extent to which a brass player can control the degree of spectral enrichment due to non-linear propagation, without significantly changing the amplitude of the sound. One option is to increase the length of the instrument tube: on a trombone, for example, the note F3 can be played with the slide in first position (fully retracted) or 6th position (almost fully extended). In the second case, the longer propagation length allows the non-linear steepening to develop further, and in addition the increased proportion of cylindrical tubing reduces the co-ordinate stretching function. The note can therefore be played with a more brassy timbre in sixth position than in first position at a given dynamic level.

Some players claim to be able to exercise this type of timbral variation without altering the tube length. The widely respected horn player and teacher Philip Farkas, for example, suggests that a brassy tone can be achieved by adopting a more 'smiling' embouchure rather than a 'whistling' one for less brassy playing [12]. Inspection of equation 4 shows that timbral change such as this could be achieved if the player were able to modify the mouthpiece waveform so as to increase the steepness of the rising edge, without altering its amplitude. The experiments and simulations described in the following sections were designed to test the hypothesis that this is indeed the mechanism used by players.

It is important to recognise that a modification of the mouthpiece waveform could well have a direct effect on the timbre of the radiated sound without taking non-linear propagation into effect. If the rising edge of the pressure waveform is steepened, it is likely that the mouthpiece spectrum will have an increased proportion of upper harmonics; the 
linear pressure transfer function will accentuate this effect through the treble boost implied by Equation 2. It was therefore necessary to design the experiments and simulations to be able to separate the linear and non-linear contributions to the spectral enrichment.

It is worth noting however that the non-linear steepening depends on the form of the time domain signal in the mouthpiece rather than the mouthpiece frequency spectrum. The two pressure waveforms illustrated in Figure 2 have the same frequency spectra, but the upper wave would develop into a shock wave in a much shorter propagation distance than the lower wave.

\section{$3 \quad$ Experimental procedure and results}

The experimental set-up for examining the relationship between sound pressure in the mouthpiece and that of the radiated sound from the bell of the instrument is shown in Figure 3. The pressure in the mouthpiece of the instrument was measured using a 106B PCB Piezotronics dynamic pressure transducer. A commercially available horn mouthpiece, a Paxman-Halstead-Chidell (PHC 22), was modified to accommodate the PCB microphone into the backbore of the mouthpiece as shown in Figure 4. The radiated sound from the bell of the instrument was measured using a Bruël and Kjær 4192 pressurefield microphone, located one bell diameter from the plane of the bell of the instrument. The signals from both transducers were sampled at $44.1 \mathrm{kHz}$.

A total of six professional and skilled amateur horn players were asked to provide playing samples. The instrument used for the tests was a Meinl \& Lauber/Paxman 'baroque' horn modelled after an 18th-century instrument by Huschauer, Vienna c.1770. The instrument was crooked in $\mathrm{D}$ with a tube length corresponding to a nominal pitch of $\mathrm{D}_{1}$ (approx. $4.4 \mathrm{~m}$ ). The players were also invited to use their own instruments if they wished. However, the Meinl \& Lauber/Paxman was judged to be most amenable to player control of brassiness, and the results presented are from this instrument.

The players were given a familiarisation period with the instrument before recording the test data. For the tests, the players could either sound pairs of notes, approximately 1-2 seconds each in duration, both at the same dynamic level, but one brassy and the other non-brassy; or the player could sound a single note (2 or more seconds in duration) at constant dynamic level, and change the timbre of the note as it was played. The pitch $\mathrm{D}_{3}$ was chosen as the reference for the experiments. This is a mid-range note for the horn in $\mathrm{D}$ and was found to be a comfortable pitch for players to manipulate the timbre using only the embouchure. Tests were performed with the hand supporting the bell of the instrument as is usual practice and most comfortable for horn players, and also without the hand in the bell.

To assist the players with keeping the sound at constant pitch and dynamic level they were provided with a sound-level meter (set to A-weighting) and a digital tuner. The dynamic level at which the players found that the timbre could most easily be controlled

was, in musical terms, forte, but not fortissimo. This varied marginally from player to player but was normally in the range 96-100 dB with the sound level meter positioned 
one metre in front of the player as shown in Figure 3. For the results presented here, players were able to maintain the sound pressure level to within $\pm 1 \mathrm{~dB}$ of their chosen

target. Some players also found it useful to watch the pressure signals, sampled in the mouthpiece and at the bell, on an oscilloscope whilst playing.

\subsection{Results}

Figure 5 shows examples of the frequency spectra of the radiated sound for brassy and non-brassy tones, played at the same dynamic level. The increase in strength of the higher frequency harmonics in the brassy tone compared with that of the non-brassy tone can clearly be seen. It should be noted that although the difference in timbre between the displayed brassy and non-brassy tones is significant and could easily be heard by the listener, these are by no means examples of the extremes of brassy and non-brassy playing. These extremes occur at the very quietest and loudest dynamic levels where embouchure manipulation plays a much less significant role than intensity level in determining spectral content.

Figure 6 shows the peak values of the frequency spectra for brassy and non-brassy pressures measured at the bell of the instrument and the corresponding pressure measured in the mouthpiece. Close inspection of this data shows that for frequencies above about 3.5 $\mathrm{kHz}$ the difference in spectral content of the mouthpiece pressure between brassy and nonbrassy measurements is negligible. In contrast to this, the difference in spectral content of the radiated sound at higher frequencies is large, confirming that spectral enrichment is not simply occurring in the mouthpiece.

This difference is highlighted further in Figure 7 which shows the change in the magnitude of peak values between radiated non-brassy and brassy sound pressure levels; and the change in magnitude between the input (mouthpiece) pressures levels for non-brassy and brassy playing, ie the gain in going from non-brassy to brassy. Peak values are averaged over $1 \mathrm{kHz}$ bins. This graph shows that even at approximately the same dynamic level, large differences in spectral content can clearly be seen where the two curves diverge. For example, in the range 6 to $7 \mathrm{kHz}$ the pressure level in the mouthpiece decreased by $1 \mathrm{~dB}$ in the transition from non-brassy to brassy, but increased in the radiated sound by more than $16 \mathrm{~dB}$. This would suggest that for a particular instrument, non-linearity is to some extent dependent not only on the intensity of the sound produced, but also on the way in which the player is able to control and alter the input waveform.

Figures 8 and 9 show the pressure waveforms recorded in the mouthpiece for a nonbrassy and a brassy tone of pitch $\mathrm{D}_{3}$. Although the pressures measured in the mouthpiece (Figs. 8(a) and 9(a)) appear broadly similar, on close inspection it can be seen that the rising edge of the wave is steeper for the brassy tone than for the non-brassy tone. The rate of change of pressure in the mouthpiece, $d P_{i n} / d t$, is also shown in both cases. For the mouthpiece signal which produces a non-brassy tone, the gradient of the wavefront is shown to be slightly more than $10 \mathrm{MPa} / \mathrm{s}$, while that for the brassy tone displays a gradient of more than $15 \mathrm{MPa} / \mathrm{s}$. Thus the 'brassy' mouthpiece wavefront is approximately $50 \%$ steeper than that of the 'non-brassy' signal. 


\section{Discussion and comparison with simulated results}

The experimental results presented in Section 3 show that when a player manipulates the tone of the horn by embouchure control in order to make the sound brassier, a steepening of the rising edge of the mouthpiece pressure waveform occurs. This change in shape of the waveform is not a result of a change in dynamic level or pitch, as these parameters have been kept constant. Reference to Equation 4 shows that an increase of $50 \%$ in the value of $\left(d p_{\text {in }} / d t\right)_{\max }$ will lead to a reduction by the same factor in the distance to shock formation $x_{s}$. Even if a shock wave does not fully develop in the length of the instrument, the rate of wave steepening, and therefore of spectral enrichment, will be substantially increased.

Wave steepening such as this occurs more readily in instruments which have a significantly long section of narrow cylindrical tubing, which is the reason that trumpets and trombones generally sound brassier than euphoniums and bugles [3]. The latter two instruments have a greater proportion of flaring or conical tubing and this causes any build up of pressure from the preceding cylindrical section to dissipate more rapidly as the diameter of the bore increases.

The findings from these experimental results can be assessed further by exploiting a numerical simulation tool which is able take into account the non-linear propagation phenomenon in non-uniform ducts such as brass instruments by solution of the generalised Burgers' equations [9]. Using this model, the relative contribution of the non-linear propagation effects in the radiated sound can be estimated separately from the linear effects by carrying out simulations with and without the inclusion of the nonlinear term in the generalised Burgers' equations. It should be emphasised that this excerise is carried out purely to illustrate the significance of the nonlinear contribution, which is always present in high level propagation in the real world.

The frequency model simulation method is based on previous publications dedicated to uniform ducts and clarinet-like instruments [13, 14], and has been adapted here to apply to non-uniform ducts and brass instruments. The simulation can be summarized as follows: postulating the pressure spectrum $P_{\text {in }}$ at the input end, a radiated impedance boundary condition at the output end (from Causse et al [15]), and the bore geometry of the instrument, the pressure and velocity acoustic fields are first calculated everywhere inside the bore using the weakly non-linear approximation. Indeed, the interaction between the simple waves propagating in each direction is supposed to negligible, as in the linear approximation. The numerical solution to these equations, and details of the numerical harmonic balance convergence methods, are detailed in [13]. The volume velocity spectrum $Q_{\text {out }}$ at the output end of the instrument is estimated by multiplying the output area by the output acoustic velocity spectrum. By using the low frequency approximation for a monopole having a volume strength equal to $Q_{\text {out }}$, a radiated pressure spectrum and its spectral centroid are estimated at a distance $r$ from the open end of the pipe from Equation 2.

Simulations were performed using the bore geometry for the Meinl \& Lauber/Paxman horn described in Section 3, tuned to a nominal pitch $\mathrm{D}_{1}$ (horn length of approximately 4.4 
m). Two pressure spectra, $P_{\text {in }}$, at the input end are used as input data for the simulations. These are taken directly from experimental data collected in the side of the mouthpiece backbore during playing tests, as described above: one corresponding to a waveform which produces a brassy tone, the other a waveform which produces a non-brassy tone. The original input data can be seen in Figure 6, and the results of the simulations can be seen in Figure 10.

For each input data set the model was run twice: first, taking into account the nonlinear propagation effects; and second, without taking into account the non-linear effects, shown in Figure 10(a). In the first case, the displayed results exhibit a clear offset in the high frequency part of the spectral envelopes between the brassy and non-brassy tones. This offset is qualitatively comparable to that of the measured data shown in Figure 6. This offset is clearly a consequence of the non-linear propagation and not simply due to the small differences in mouthpiece spectra. Indeed, if the non-linear propagation is not taken into account in the simulations, then the offset disappears and the calculated radiated spectral envelopes are more or less the same even at high frequencies as shown in Figure 10(a).

The pressure transfer function between the radiated and the input pressures in the simulations are shown in Figure 11. It is known that differences in amplitude between the radiated spectral components and corresponding components in the mouthpiece are not constant over all dynamic levels due to increasing non-linearity as the sound intensity increases [16]. However, Figure 11 shows that even at constant dynamic levels, for different input waveforms (specifically having different $d P_{i n} / d t$ of the wave front), large differences in amplitude can clearly be seen where the two curves diverge. The 'linear limit' can also be seen in Figure 11 showing the typical high-pass filter effect of radiation.

A second simulation was performed (with and without taking into account the nonlinear propagation effects) in order to simulate pianissimo playing; the results are shown in Figure 10(b). The input data for this are synthesized from the same two measured mouthpiece pressure spectra, $P_{i n}$, from the previous simulation, globally decreased by 20 dB. Figure 10(b) shows that at this quiet dynamic there is now little or no difference in the high frequency part of the spectral envelopes between the brassy and non-brassy tones. This is not surprising since we know that at pianissimo levels, non-linear propagation is not significant.

\section{Conclusion}

The six horn players studied demonstrated a wide range of ability to modify the timbre of a note while maintaining a constant dynamic level. The player whose results were discussed in Section 3 was most consistently able to add or subtract a brassy edge to the sound of a mid-range note played at a constant forte dynamic level. Study of the mouthpiece pressure waveforms for brassy and non-brassy tones showed that the modification of embouchure used by this player in passing from non-brassy to brassy timbre resulted in a delayed onset and resulting steepening of the rising edge of the waveform. The playing technique thus 
exploited the dependence of the non-linear wave steepening on the maximum mouthpiece pressure gradient in order to control the brightness of the sound at constant dynamic level.

Simulations carried out using weak non-linear shock theory and the harmonic balance technique confirmed that, although the frequency dependence of the linear transfer function from internal to radiated pressure did contribute significantly to the change in timbre, the major factor in the spectral enrichment was non-linear propagation and wave steepening.

Although some players have observed that it is possible to control onset of brassiness through embouchure manipulation, the exact mechanism used by the player to modify the mouthpiece pressure waveform has not yet been identified. It is likely that manipulation of the embouchure, including a combination of changes to the mechanical parameters of the lip valve and adjustments to the acoustical parameters of the mouth cavity are involved. Studies using a transparent mouthpiece and a high speed digital camera may help to explain how some brass instrument players are able to exercise this subtle but musically important form of timbral variation. 


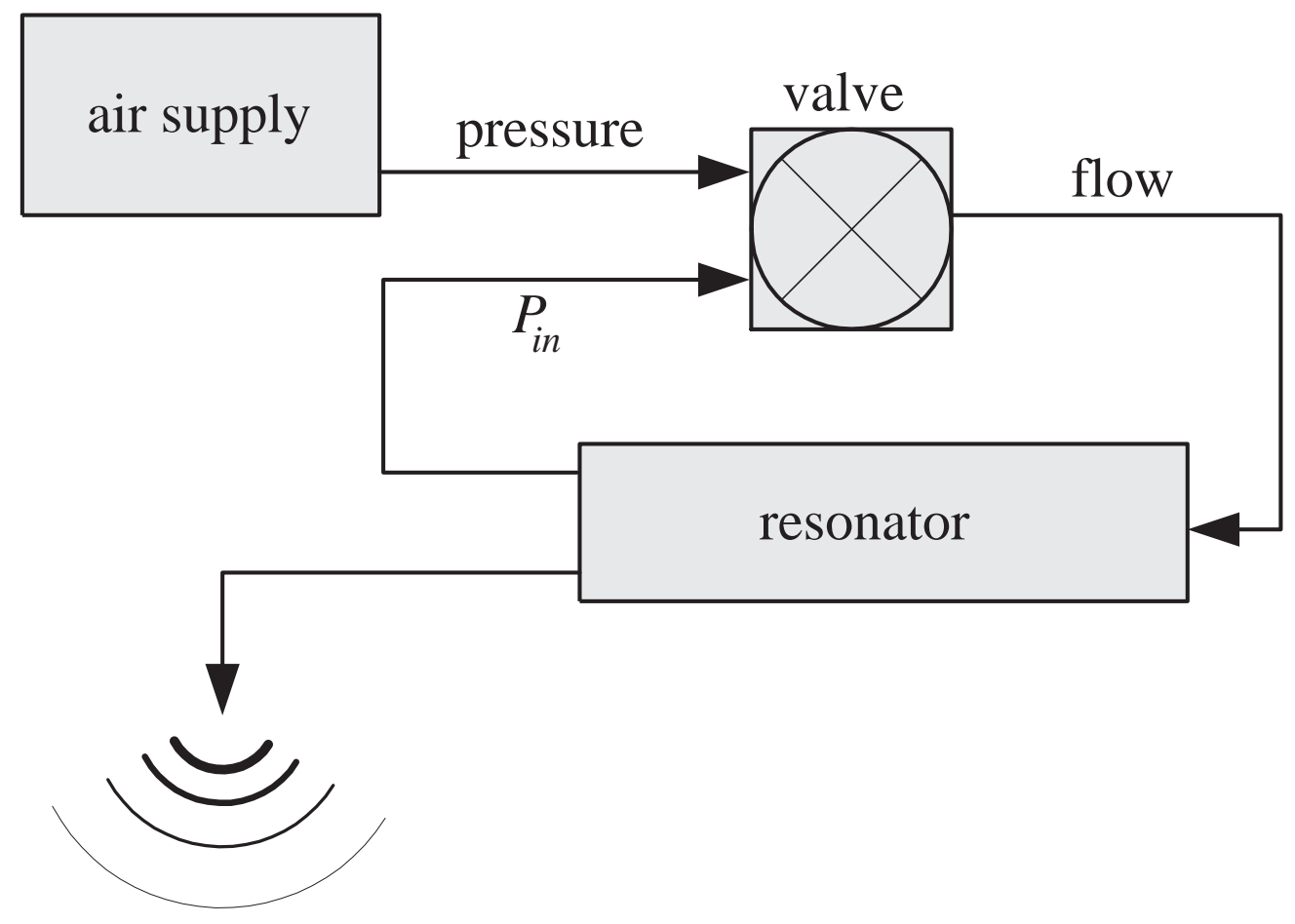

radiated sound

Figure 1: Feedback loop model of a brass wind instrument. 

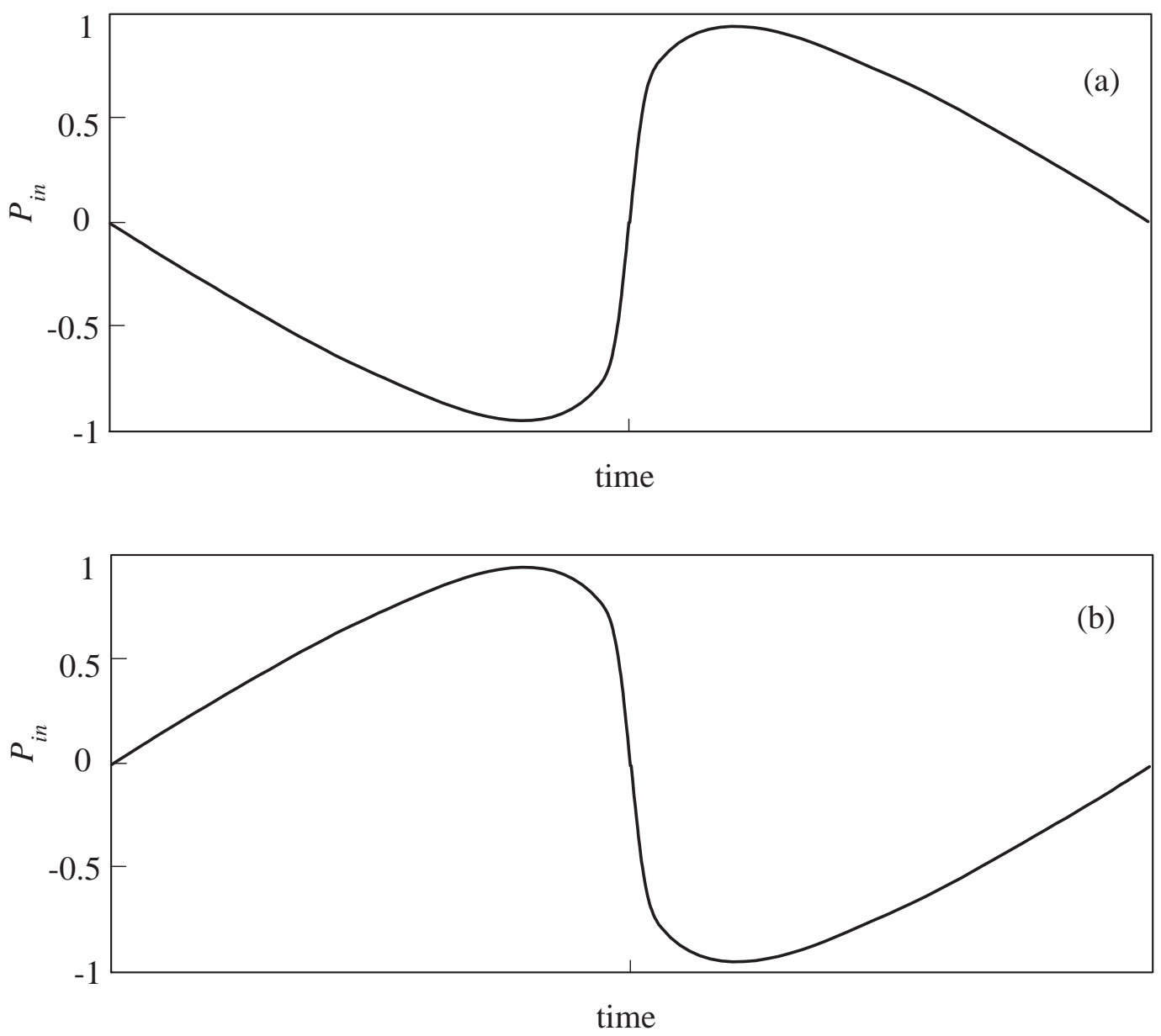

Figure 2: By changing the value of $d P_{\text {in }} / d t$ the player has some control over non-linear propagation effects even when the spectral content of the input wave is unchanged: (a) a high value of $d P_{\text {in }} / d t$ will induce large non-linear effects, (b) a low value of $d P_{i n} / d t$ will reduce non-linear effects. 


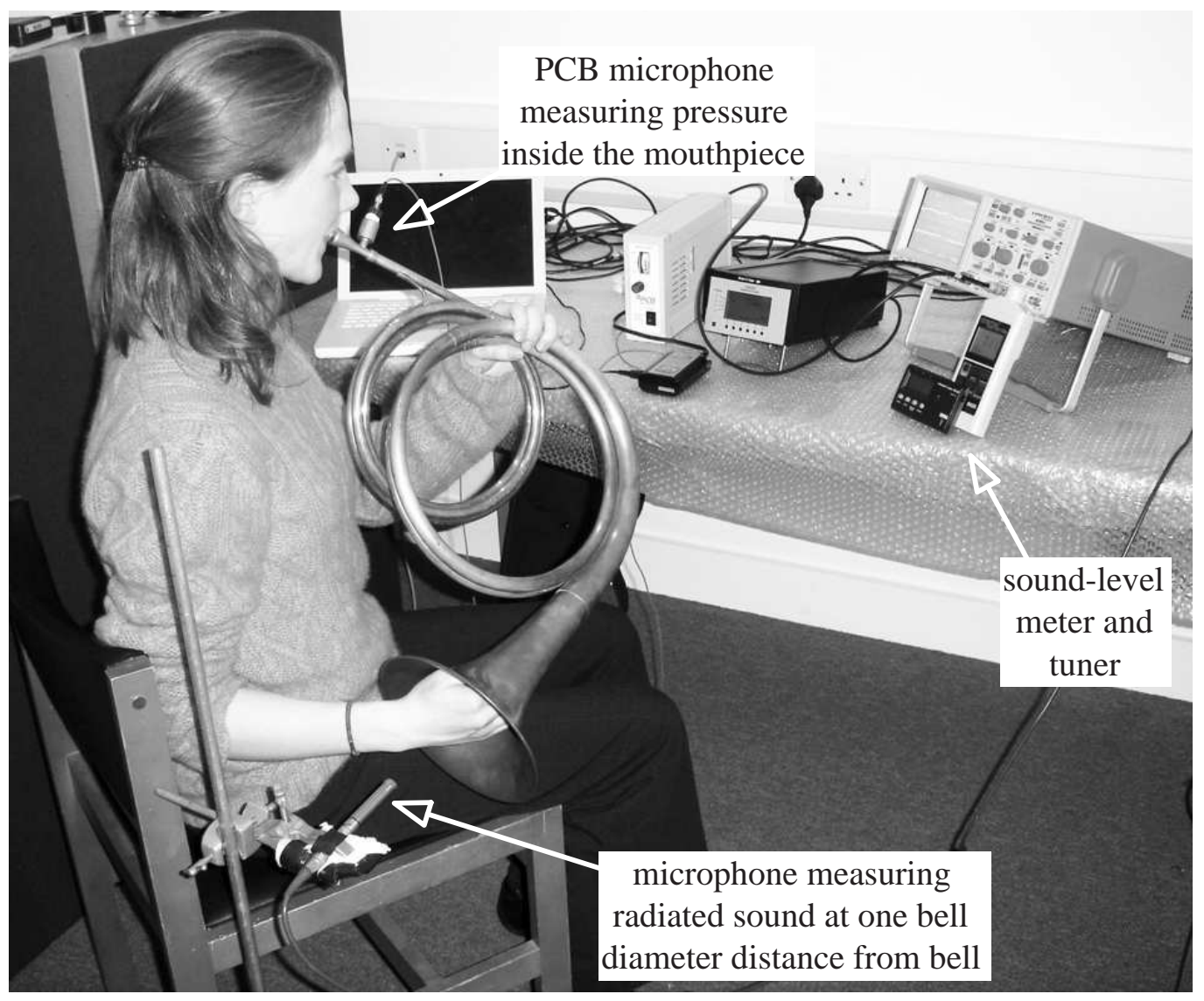

Figure 3: Experimental set up for embouchure control of brassiness playing, showing the positions of the microphones and sound-level meter. 


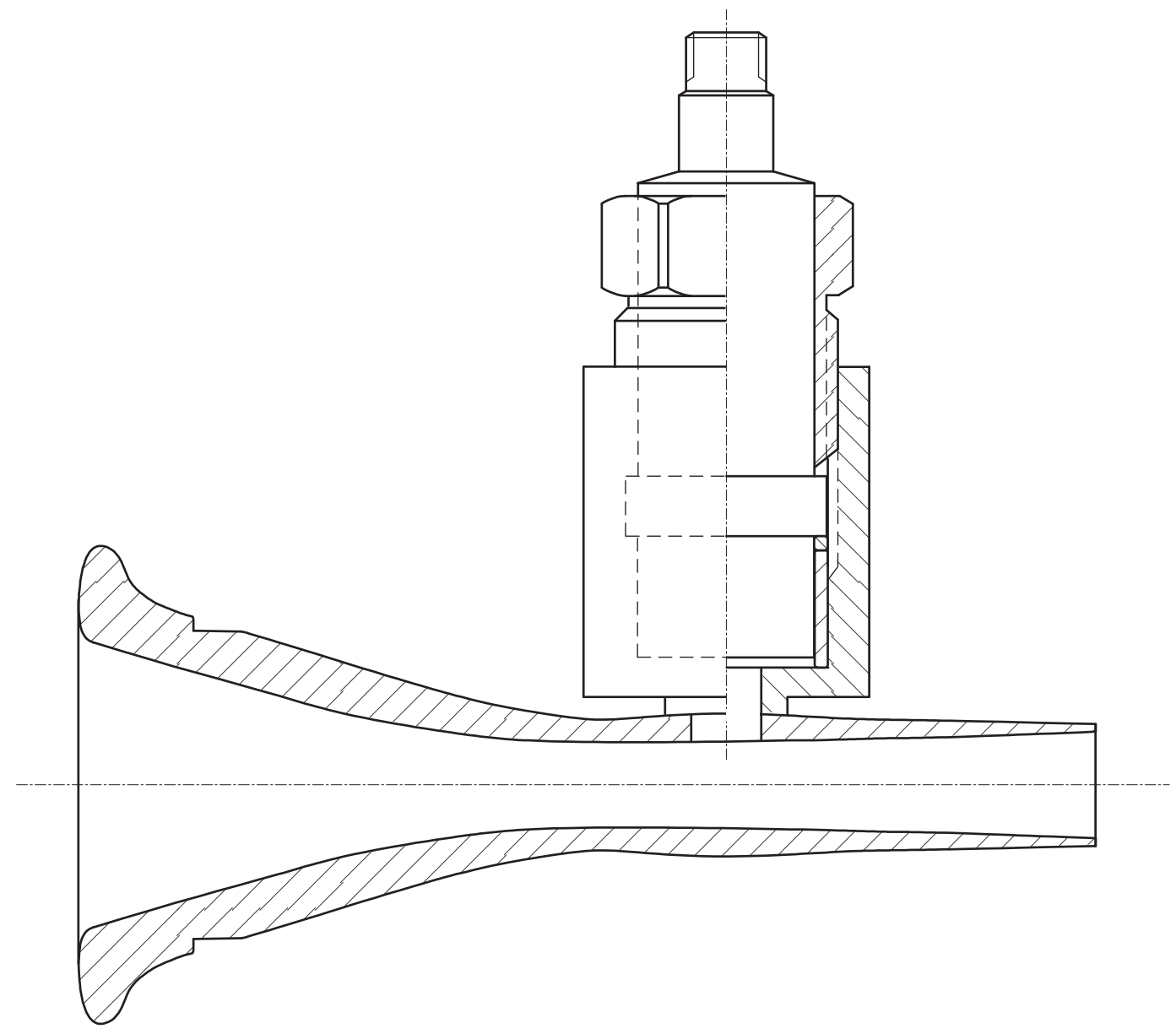

Figure 4: Horn mouthpiece and microphone assembly. 


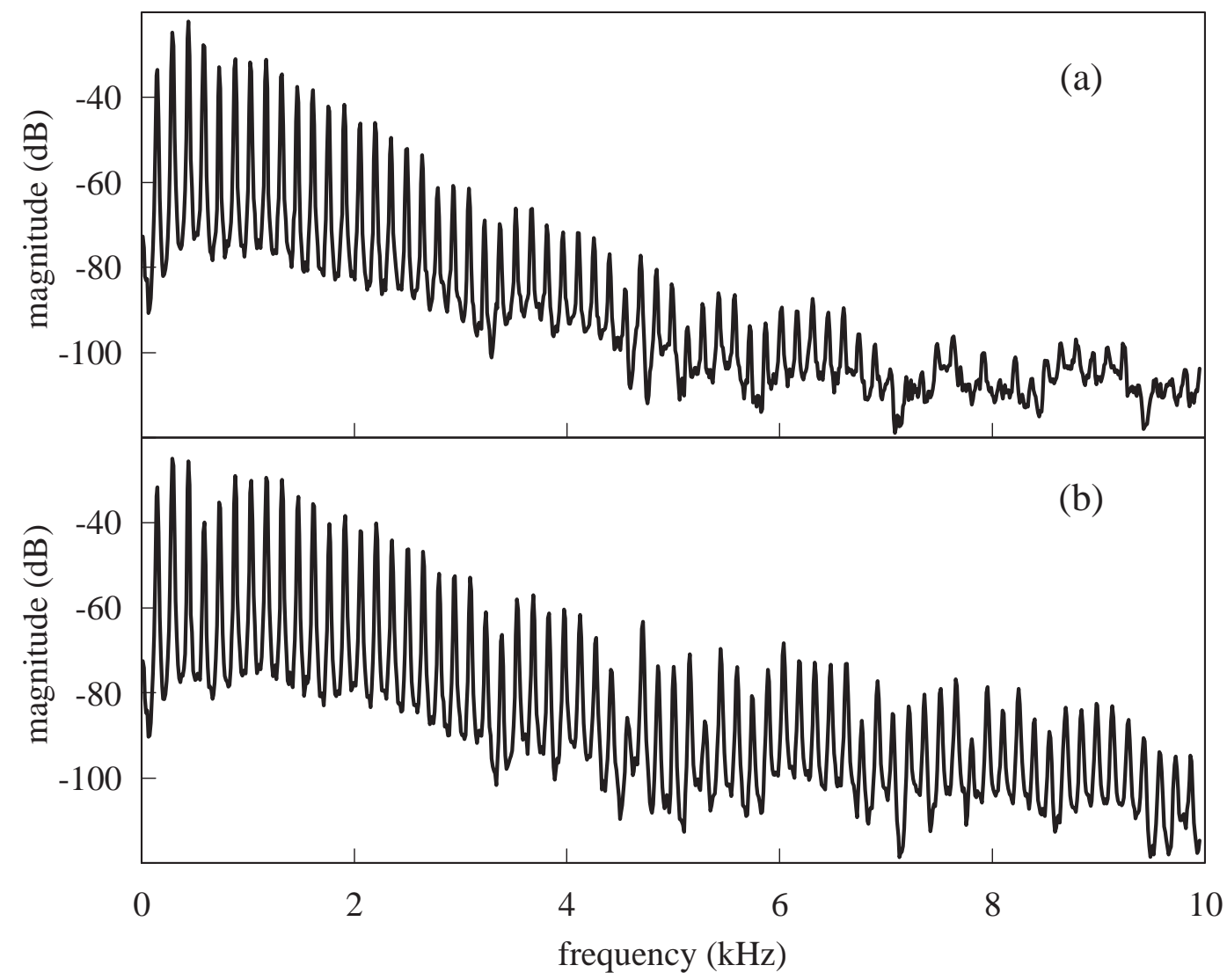

Figure 5: Frequency spectra of the radiated sound measured at one bell diameter from the bell exit for the note $\mathrm{D}_{3}$, for a non-brassy tone (a), and a brassy tone (b). Both tests were played at nominally the same dynamic level. 


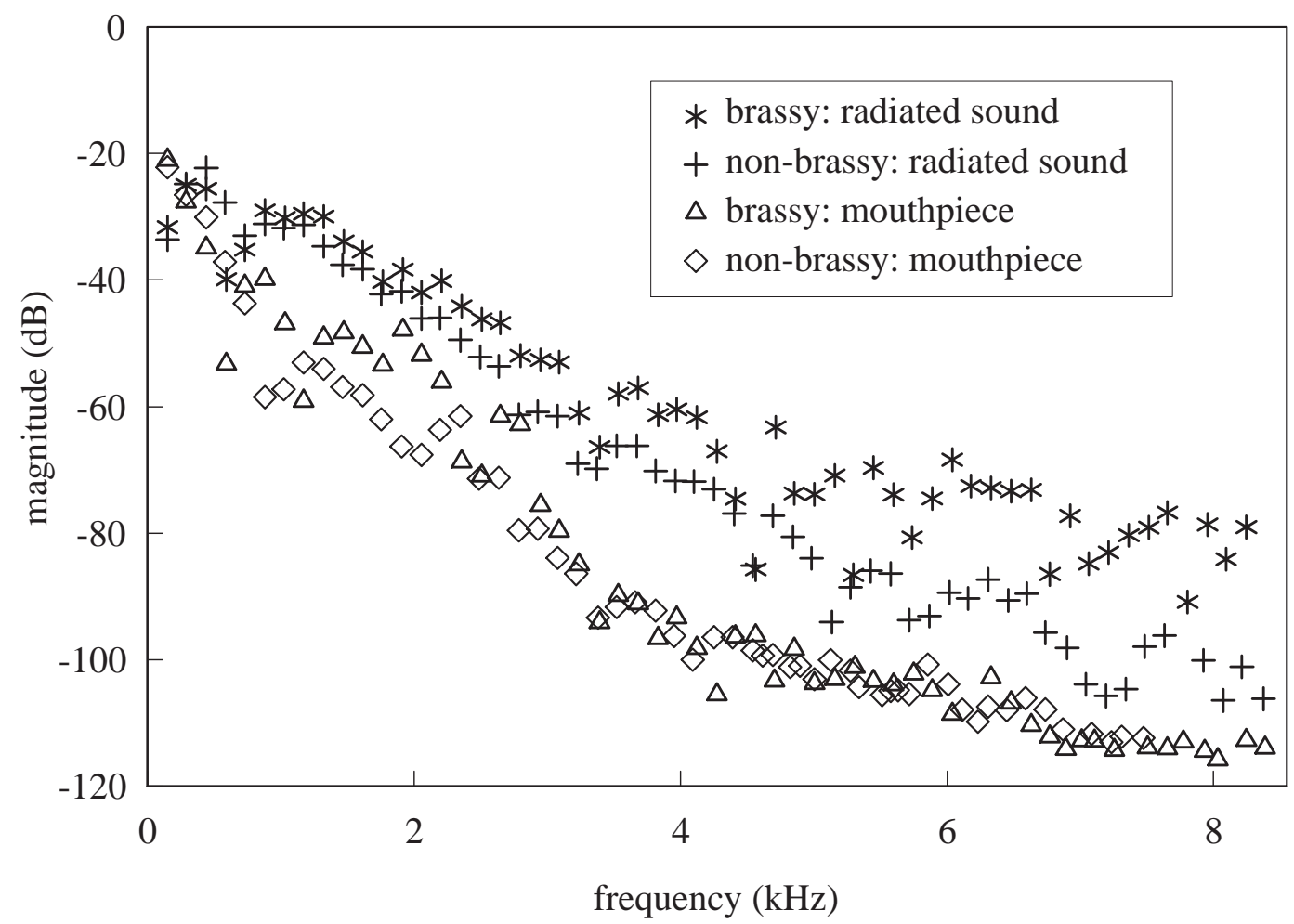

Figure 6: Spectral envelopes of brassy and non-brassy tones from mouthpiece and radiated sound. Notes $\mathrm{D}_{3}$ played at constant dynamic level on a $4.4 \mathrm{~m}$ horn in $\mathrm{D}$. 


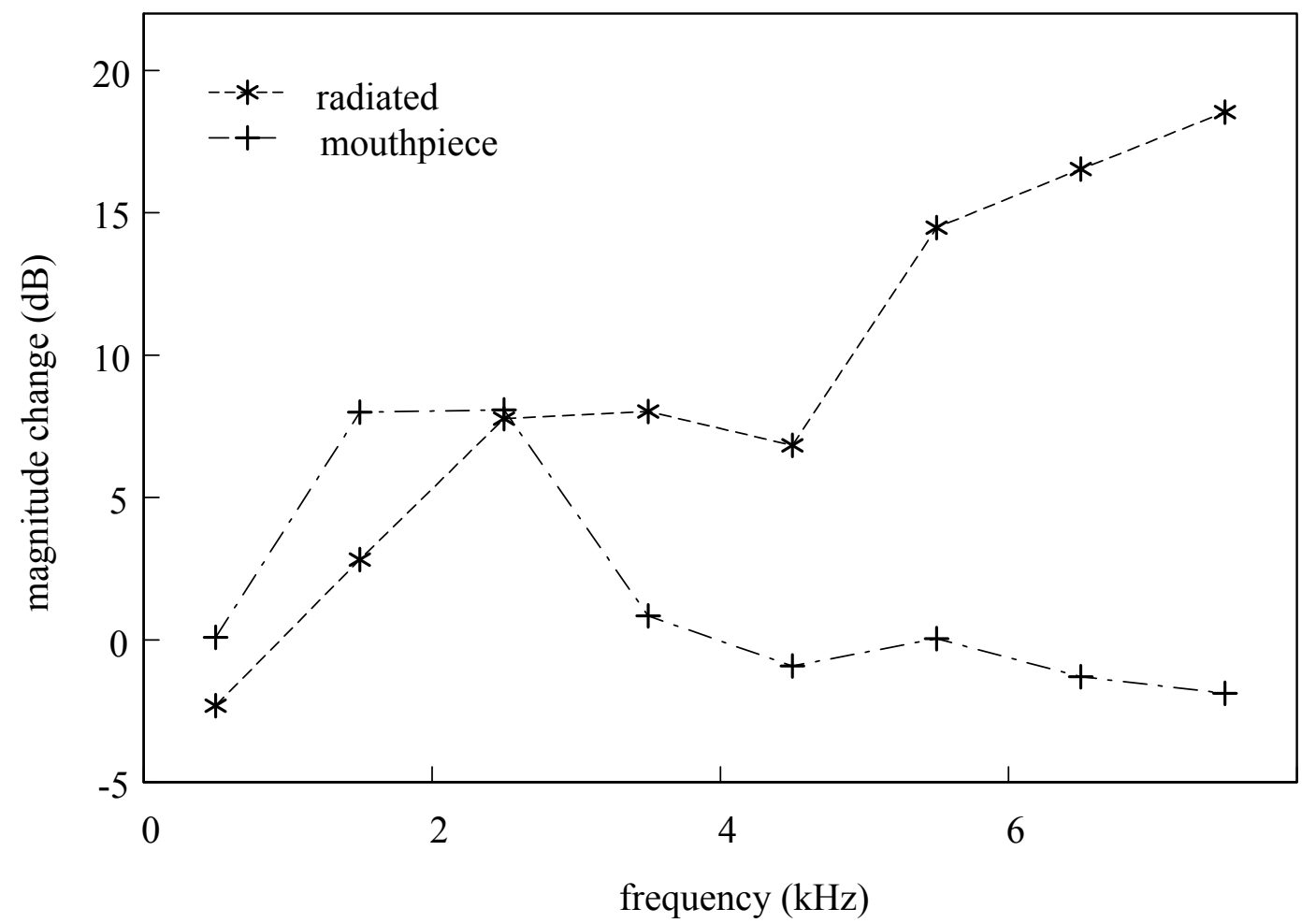

Figure 7: Change in the magnitude of peak values going from a non-brassy to a brassy sound for radiated and mouthpiece pressures. Notes $\mathrm{D}_{3}$ played at constant dynamic level on a $4.4 \mathrm{~m}$ horn in D. Peak values are averaged over $1 \mathrm{kHz}$ bins. 


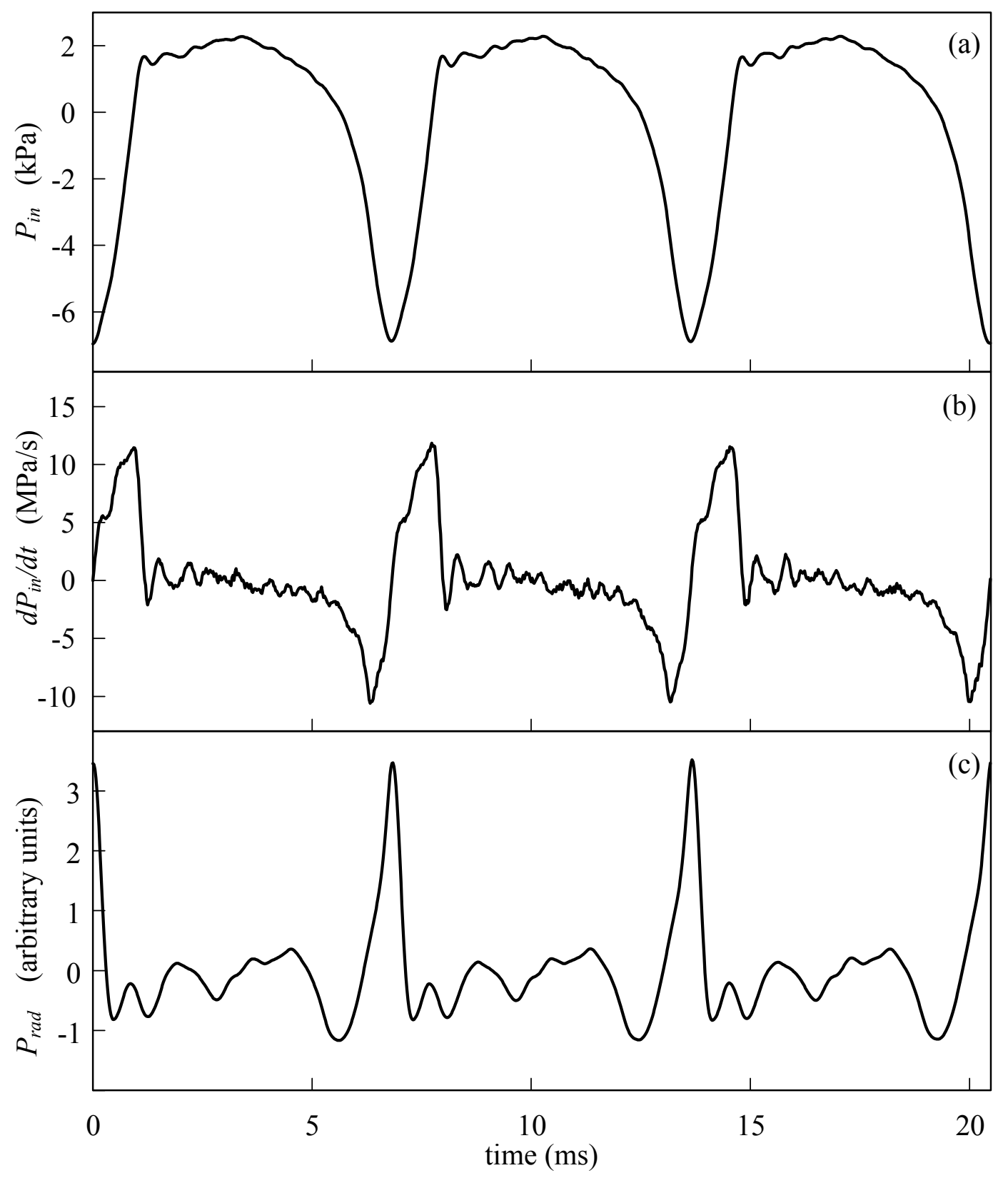

Figure 8: Measured data from non-brassy tests for the note $\mathrm{D}_{3}$. (a) shows the mouthpiece pressure waveform; (b) shows the rate of change of mouthpiece pressure; (c) shows the radiated pressure waveform. 


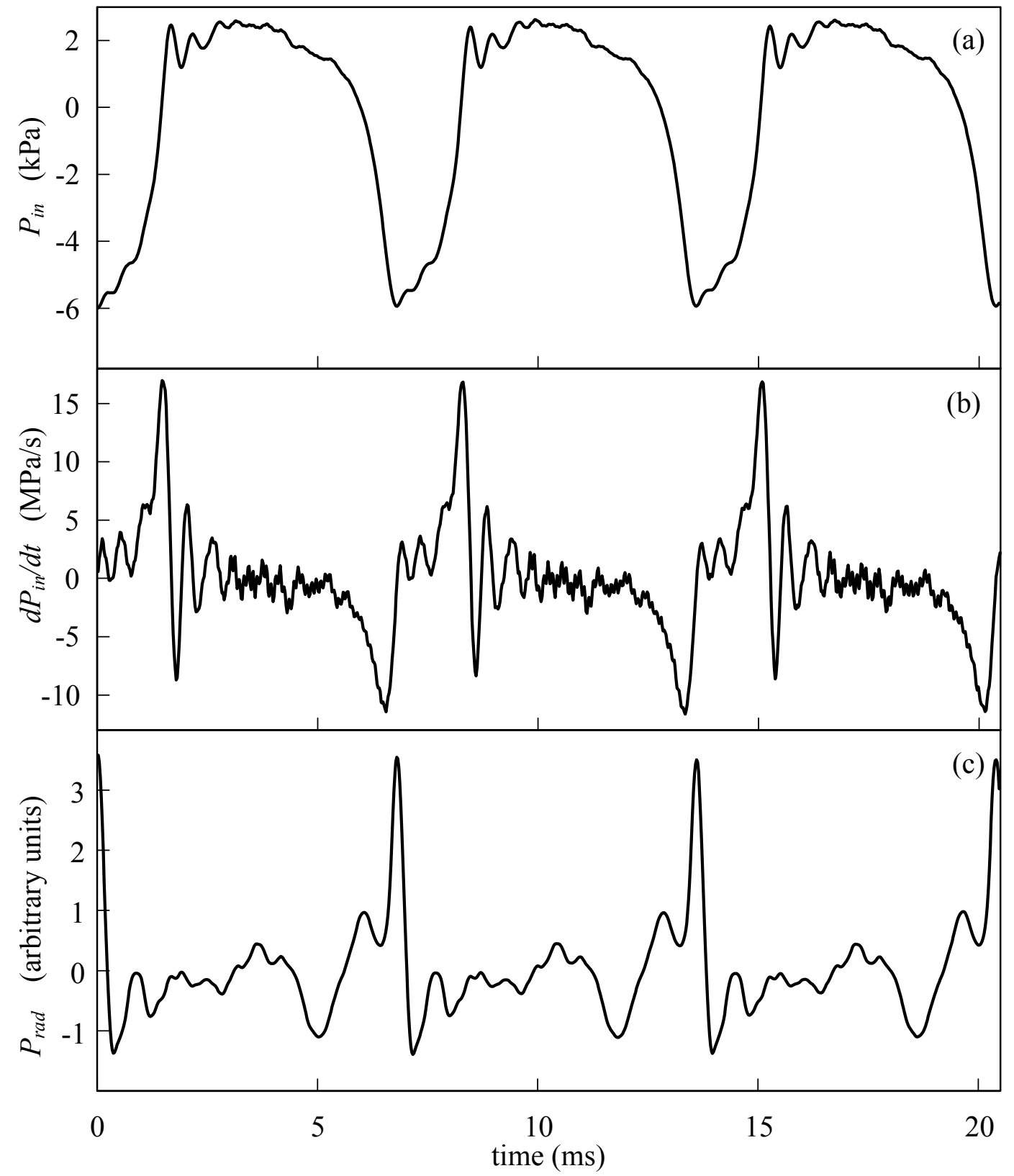

Figure 9: Measured data from brassy tests for the note $D_{3}$. (a) shows the mouthpiece pressure waveform; (b) shows the rate of change of mouthpiece pressure; (c) shows the radiated pressure waveform. 

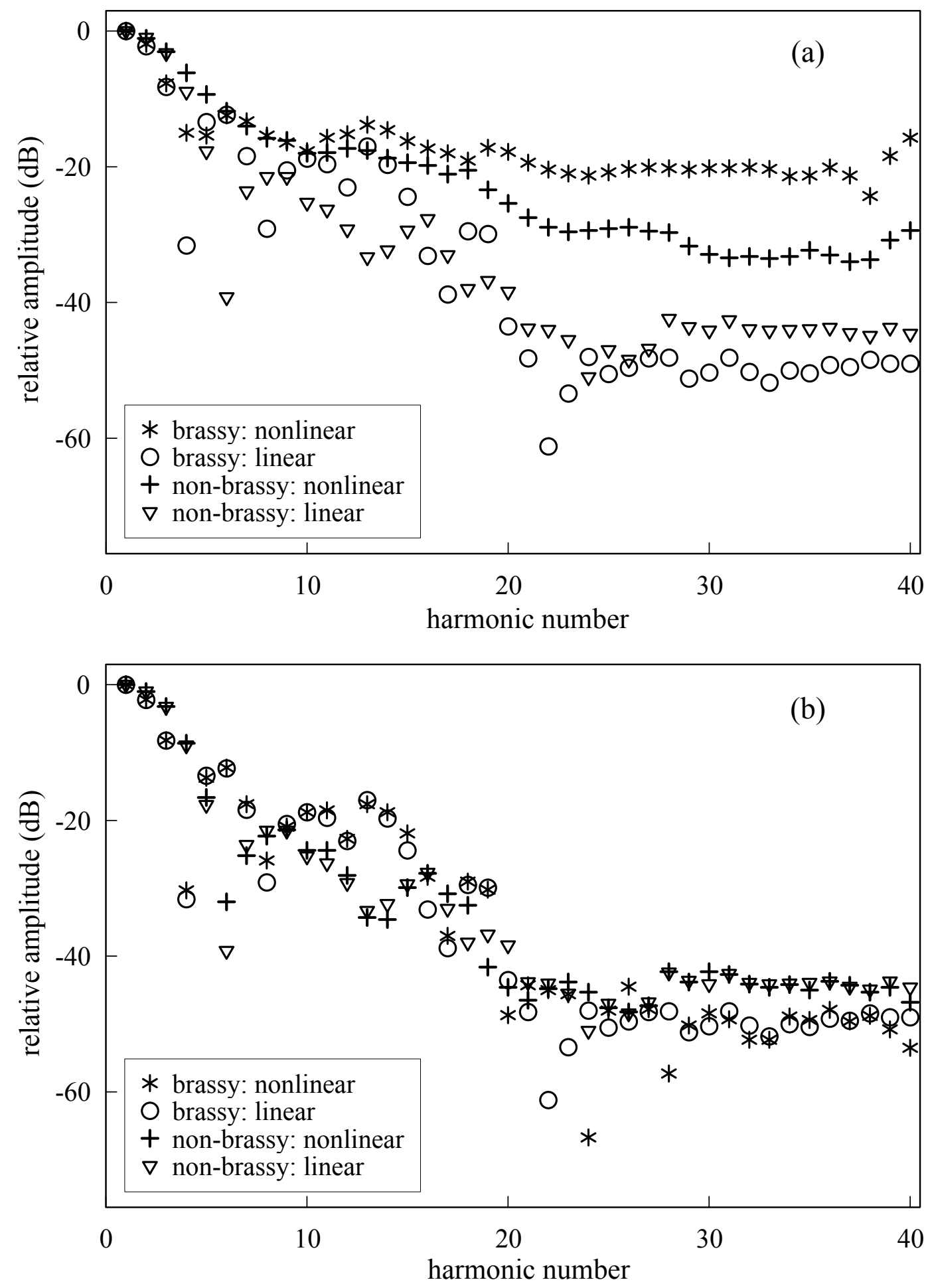

Figure 10: Spectral envelopes of brassy and non-brassy tones (amplitudes shown are relative to the fundamental, in $\mathrm{dB}$ ): (a) modelled values of radiated sound with and without non-linear propagation; (b) modelled values of radiated sound at pianissimo dynamic, where the pianissimo input is synthesized by decreasing the levels used in the previous simulation by $20 \mathrm{~dB}$. 


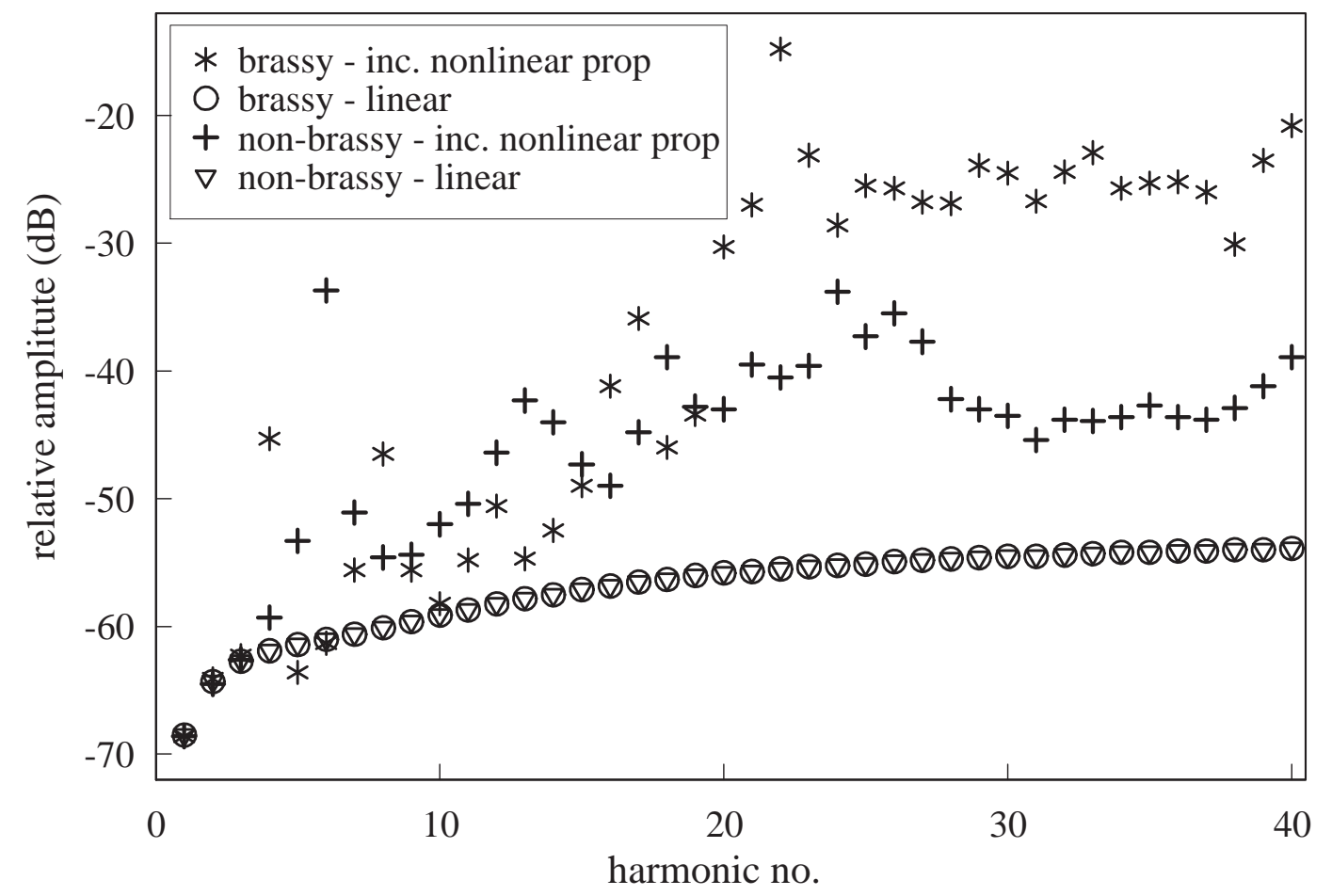

Figure 11: Transfer function, $P_{r a d} / P_{i n}$, corresponding to brassy and non-brassy tones from simulations with and without non-linear propagation. 


\section{References}

[1] A. Hirschberg, J. Gilbert, R. Msallam, and A.J.P. Wijnands. Shock waves in trombones. J. Acoust. Soc. Am., 99(3):1754-1758, March 1996.

[2] J. Gilbert, D.M. Campbell, A. Myers, and R.W. Pyle. Differences between brass instruments arising from variations in brassiness due to non linear propagation. In Proceedings of International Symposium on Musical Acoustics, Barcelona, 2007.

[3] A. Myers, J. Gilbert, R.W. Pyle, and D.M Campbell. Non-linear propagation characteristics in the evolution of brass musical instrument design. In Proceedings, 19th International Congress on Acoustics, Madrid, 2007.

[4] E. Poirson, J-F. Petiot, and J. Gilbert. Study of the brightness of trumpet tones. J. Acoust. Soc. Am., 118(4):2656-66, October 2005.

[5] R.W. Pyle. The effect of wall materials on the timbre of brass instruments. In Proc. 16th International Congress on Acoustics and 135th JASA Meeting, volume 3, pages 751-752, Seattle, 1998.

[6] R.W. Pyle. Does a brass-instrument's timbre depend on the alloy from which it is made? In 135th JASA Meeting, Portland, April 2009.

[7] D.M. Campbell and C.A Greated. The Musician's Guide to Acoustics. Oxford University Press, New York, 1987.

[8] D.M. Campbell. Brass instruments as we know them today. Acta Acustica united with Acustica, 90(4):600-610, 2004.

[9] J. Gilbert, L. Menguy, and D.M. Campbell. A simulation tool for brassiness studies. J. Acoust. Soc. Am., 123(4):1854-1857, April 2008.

[10] A.H. Benade. Fundamentals of musical acoustics. 2nd edition. Dover, 1990.

[11] M.F. Hamilton and D.T. Blackstock, editors. Nonlinear Acoustics. Academic Press, New York, 1998.

[12] P. Farkas. The Art of French Horn Playing. Summy-Birchard, 1956.

[13] J. Menguy and J. Gilbert. Weakly nonlinear gas oscillations in air-filled tubes; solutions and experiments. Acta Acustica, 86:798-810, 2000.

[14] J. Gilbert, J.P. Dalmont, and T. Guimezanes. Nonlinear propagation in woodwinds. In Proceedings of the Forum Acusticum 2005, Budapest, Hungary, 2005.

[15] R. Caussé, J. Kergomard, and X. Lurton. Input impedance of brass musical instruments-comparison between experiment and numerical models. J. Acoust. Soc. Am., 75(1):241-254, January 1984. 
[16] J.W. Beauchamp. Analysis of simultaneous mouthpiece and output waveforms. Audio Engineering Society, preprint No. 1626:1-11, 1980. 